\title{
BP-C2 (complexes of molybdenum with polymer of benzene polycarboxylic acids derived from lignin): Evaluation of antigenotoxic potential in COMET assay
}

\author{
Pigarev S.E. ${ }^{1,2}$, Trashkov A.P. $^{3}$, Anisimov V.N. ${ }^{2}$, Fedoros E.I. ${ }^{1,2}$, Panchenko A.V. ${ }^{2}$ \\ ${ }^{1}$ Nobel LTD, Saint-Petersburg, Russia, spigarev@scioco.com \\ ${ }^{2}$ Petrov National Medical Research Center of Oncology, Saint Petersburg, Russia \\ ${ }^{3}$ Petersburg Nuclear Physics Institute named by B.P. Konstantinov of NRC «Kurchatov Institute», \\ Gatchina, Russia
}

Keywords: polyphenols, comet assay, genotoxicity, safety

doi: 10.36291/HIT.2019.pigarev.097

BP-C2 is a drug candidate developed as a radiomitigator for civic applications and as a medical countermeasure for radiation emergencies [1]. In rodents exposed to gammaradiation BP-C2 has demonstrated radiomitigative potential believed to be mediated through hematopoietic and intestinal progenitor stem cells [2].

Objective of the study presented here was to evaluate antigenotoxic potential of BPC2 using in-vivo alkaline COMET assay. 35 male albino rats (Rattus norvegicus) were randomized to one of the 5 experimental groups $(n=7) .0 .9 \%$ sodium chloride was used as the negative and methyl methanesulfonate (MMS) as the positive control. MMS (40 mg/kg) or $\mathrm{NaCl} 0.9 \%$ were administered intraperitoneally once. BP-C2 was administered at 20 $\mathrm{mg} / \mathrm{kg}, 200 \mathrm{mg} / \mathrm{kg}$ or $2000 \mathrm{mg} / \mathrm{kg}$ by gavage once 1 hour before administration of MMS.

Effects of MMS and BP-C2 were assessed by estimating the percentage of DNA in comet tail in three cell subpopulations: hepatocytes, red bone marrow cells and peripheral blood leucocytes (Table 1).

Table 1. Amount of DNA in the comet tail, \%

\begin{tabular}{llllccc}
\hline \multirow{2}{*}{ Target } & Time & $\begin{array}{c}\text { NaCl 0.9\% } \\
\text { (Negative } \\
\text { control) }\end{array}$ & $\begin{array}{c}\text { MMS } \\
\text { (Positive } \\
\text { control) }\end{array}$ & $\begin{array}{c}\text { MMS+BPC2 } \\
20 \mathrm{mg} / \mathrm{kg}\end{array}$ & $\begin{array}{c}\text { MMS+BPC2 } \\
100 \mathrm{mg} / \mathrm{kg}\end{array}$ & $\begin{array}{c}\text { MMS+BPC2 } \\
2000 \mathrm{mg} / \mathrm{kg}\end{array}$ \\
\hline \multirow{3}{*}{ Hepatocytes } & $1 \mathrm{hr}$. & $5.5 \pm 0.8$ & $14.2 \pm 3.6^{1}$ & $11.1 \pm 1.5^{1}$ & $9.8 \pm 1.0^{1,2}$ & $7.9 \pm 1.3^{2}$ \\
\cline { 2 - 7 } & $6 \mathrm{hrs}$. & $6.8 \pm 0.8$ & $24.9 \pm 4.0^{1}$ & $18.1 \pm 3.2^{1}$ & $9.0 \pm 0.1^{1,2}$ & $8.1 \pm 1.7^{2}$ \\
\cline { 2 - 7 } & $18 \mathrm{hrs}$. & $5.6 \pm 0.7$ & $31.3 \pm 5.2^{1}$ & $17.9 \pm 4.0^{1,2}$ & $10.2 \pm 1.4^{1,2}$ & $9.0 \pm 3.0^{2}$ \\
\hline \multirow{3}{*}{ Leucocytes } & $1 \mathrm{hr}$. & $3.8 \pm 0.7$ & $8.8 \pm 1.0^{1}$ & $8.0 \pm 1.0^{1}$ & $4.6 \pm 1.4^{2}$ & $5.0 \pm 0.8^{2}$ \\
\cline { 2 - 7 } & $6 \mathrm{hrs}$. & $3.1 \pm 0.6$ & $13.5 \pm 1.0^{1}$ & $15.5 \pm 2.0^{1}$ & $3.8 \pm 0.6^{2}$ & $9.7 \pm 2.0^{1}$ \\
\cline { 2 - 7 } Red bone & $18 \mathrm{hrs}$. & $4.0 \pm 0.6$ & $17.2 \pm 2.8^{1}$ & $13.5 \pm 2.0^{1}$ & $5.1 \pm 2.3^{2}$ & $10.3 \pm 1.1^{1,2}$ \\
\hline \multirow{2}{*}{ marrow cells } & $1 \mathrm{hr}$. & $11.9 \pm 2.4$ & $23.3 \pm 3.4^{1}$ & $19.1 \pm 3.4^{1}$ & $18.8 \pm 6.6^{1}$ & $19.3 \pm 2.0^{1}$ \\
\cline { 2 - 7 } & $6 \mathrm{hrs}$. & $9.8 \pm 1.4$ & $33.1 \pm 2.5^{1}$ & $25.1 \pm 3.3^{1,2}$ & $19.5 \pm 3.2^{1,2}$ & $21.0 \pm 2.9^{1,2}$ \\
\cline { 2 - 7 } & $18 \mathrm{hrs}$. & $8.1 \pm 1.5$ & $30.7 \pm 1.9^{1}$ & $20.4 \pm 2.5^{1,2}$ & $13.5 \pm 1.4^{1,2}$ & $10.1 \pm 1.2^{2}$ \\
\hline
\end{tabular}

${ }^{1}-p<0.05$ vs Control; ${ }^{2}-p<0.05$ vs MMS

In animals treated with MMS, at the lowest tested dose (20 mg/kg) BP-C2 significantly reduces percentage of DNA in comet tail in all three cell types, though not reversing it to the level observed in the control group. At the higher doses (100 or 2000 $\mathrm{mg} / \mathrm{kg}$ ) BP-C2 almost completely reverses DNA damage in all three cell types.

\section{References}

1. Anisimov V.N. et al. // Oncotarget. 2017. 8(59):100951-100956.

2. Bykov V.N. et al. // Int J Radiat Biol. 2018. 94(2):114-123. 